\title{
NECESSARY AND SUFFICIENT CONDITIONS FOR DETERMINING A HILL'S EQUATION FROM ITS SPECTRUM ${ }^{1}$
}

\author{
BY WALLACE GOLDBERG ${ }^{2}$ \\ Communicated by Eugene Isaacson, October 24, 1974
}

A Hill's equation is an equation of the form

$$
y^{\prime \prime}+[\lambda-q(z)] y=0 .
$$

We assume $q(z+\pi)=q(z)$, where $q(z)$ is integrable over $[0, \pi]$. Without loss of generality, it is customary to assume that $\int_{0}^{\pi} q(z) d z=0$. The discriminant of (1) is defined by $\Delta(\lambda)=y_{1}(\pi)+y_{2}^{\prime}(\pi)$ where $y_{1}$ and $y_{2}$ are solutions of (1) satisfying $y_{1}(0)=y_{2}^{\prime}(0)=1$ and $y_{1}^{\prime}(0)=y_{2}(0)=0$.

The set of values of $\lambda$ for which $|\Delta|>2$ consists of a finite or an infinite number of finite disjoint intervals and one infinite interval. These intervals are called instability intervals, since (1) has no solution which is bounded for all real $z$ in these intervals. When $|\Delta|<2$, all solutions of (1) are bounded for all real $z$ and the corresponding intervals are called stability intervals. Pertinent information about stability and instability intervals of (1) can be found in Magnus and Winkler [1].

In [2] it was proved that if $q(z)$ is real and integrable, and if precisely $n$ finite instability intervals fail to vanish, then $q(z)$ must satisfy a differential equation of the form

$$
q^{(2 n)}+H\left(q, q^{\prime}, \cdots, q^{(2 n-2)}\right)=0
$$

where $H$ is a polynomial of maximal degree $n+2$. Explicit expressions of this equation are displayed in [2] and [3] for the cases $n=0,1$ and 2 .

For an infinite class of Korteweg-deVries equations of the form

$$
q_{t}=K_{n}\left(q, q_{z}, \cdots, \partial^{2 n+1} q / \partial z^{2 n+1}\right) \quad(n=0,1,2, \cdots),
$$

AMS (MOS) subject classifications (1970). Primary 34B30; Secondary 34E05.

1 This research was supported by the City University of New York Faculty Research Award Program \#10617.

2 I thank Harry Hochstadt for his generous advice during numerous consultations. 
Lax [4] has found that a periodic function $q$ satisfying

$$
K_{n}\left(q, \cdots, \partial^{2 n+1} q / \partial z^{2 n+1}\right)=0
$$

requires (1) to have no more than $n$ finite instability intervals. Explicit computations in [2] have shown (2) and (3) to be equivalent for the cases $n=0,1$ and 2. These results have now been extended to show that (2) and (3) are equivalent for all values of $n$. Hence we have necessary and sufficient conditions which the periodic potential function $q(z)$ must satisfy when $n$ finite instability intervals of (1) fail to vanish.

The proof of this result is accomplished by comparing an asymptotic expression of the solution of the related problem

$$
\left\{\begin{array}{l}
u^{\prime \prime}+[\lambda-q(z+t)] u=0 ; t \text { real, arbitrary } \\
u(0)=0, u^{\prime}(0)=1
\end{array}\right.
$$

at $z=\pi$ with $y_{2}(\pi)$ and by assuming Hochstadt's result [5] that $q(z)$ is infinitely differentiable when $n$ finite instability intervals fail to vanish. The details will appear in a later publication.

\section{REFERENCES}

1. W. Magnus and S. Winkler, Hill's equation, Interscience Tracts in Pure and Appl. Math., no. 20, Interscience, New York, 1966. MR 33 \#5991.

2. W. Goldberg, On the determination of a Hill's equation from its spectrum, J. Math. Anal. Appl. (to appear).

3. - On the determination of a Hill's equation from its spectrum, Bull. Amer. Math. Soc. 80 (1974), 1111-1112.

4. P. Lax, Personal communication.

5. H. Hoschstadt, On the determination of a Hill's equation from its spectrum, Arch. Rational Mech. Anal. 19 (1965), 353-362. MR 31 \#6019.

DEPARTMENT OF MATHEMATICS, QUEENS COLLEGE, FLUSHING, NEW YORK 11367 